\section{Hip protectors for preventing hip fractures in older people}

Fractures of the proximal femur (hip fractures) occur predominantly in older populations, with an average age around 80 years. Around $80 \%$ of people sustaining hip fractures are female and many are frail with other medical conditions. Between $12 \%$ and $27 \%$ of people sustaining a hip fracture die within a year, often as a direct consequence of their fracture. Most survivors fail to regain their former levels of mobility, many becoming more dependent including requiring institutional care. Worldwide, an estimated 1.7 million hip fractures occurred in 1990 with a predicted increase to 6.29 million by the year 2050. The already immense burden on society is increasing, including the escalating costs of the additional institutional care for many people. Preventing these injuries is a clear public health priority.

Hip fractures generally result from a simple fall, from standing height or less, in bone weakened by osteoporosis. Loss of protective mechanisms, such as putting out the arms to break the fall, in old people means that the main impact with the ground is usually on the side in the region of the hip. External hip protectors, acting to reduce the direct impact of a fall, have been promoted as a means of preventing hip fractures. The two key types of hip protector are hard protectors generally in the form of plastic shields, which act to deflect the force away from the greater trochanter (a bony prominence of the femur) to the surrounding soft tissues, and soft protectors, such as foam pads, which are energy absorbing. Both types of device are usually fitted in pockets within specially designed underwear. Potentially, hip protectors could be one way of reducing the burden of hip fracture, but do they work? This is the subject of a recently published update of a review in the Cochrane Database of Systematic Reviews that examines the accumulating evidence on whether the wearing of hip protectors reduces the incidence of hip fractures in older people following a fall. ${ }^{1}$

\section{HIP PROTECTORS FOR PREVENTING HIP FRACTURES IN OLDER PEOPLE}

This review ${ }^{1}$ by Gillespie et al includes 16 randomised controlled trials involving a total of over 16000 older people. The findings of 13 trials conducted in nursing or residential care settings were reported separately from the three trials involving older people living in the community. In seven institutional trials, the participating facilities were cluster randomised to the provision of hip protectors or not. All three community trials used hard protectors, as did nine institutional trials.

The review found that data pooled from the 13 trials (11573 participants) conducted in nursing or residential care settings showed a marginally significant reduction in hip fracture risk (RR $0.81,95 \%$ CI 0.66 to 0.99 ). However, a sensitivity analysis entailing the removal of data from the five studies at high risk of selection bias resulted in a reduced effect size and loss of statistical significance (RR $0.93,95 \%$ CI 0.74 to 1.18). From this evidence, the review's authors concluded that, while hip protectors may reduce hip fractures in these settings, their effectiveness remains uncertain. The review authors found no evidence that provision of hip protectors significantly changed the incidence of pelvic fracture, other fractures, or falls.

Pooling of data from the three community trials (5135 participants) showed no evidence of reduction in hip fracture risk (RR 1.14, 95\% CI 0.83 to 1.57 ).

For hip protectors to be effective, people need to agree to wear them (acceptance) ${ }^{2}$ and to continue wearing them in a correct manner (adherence). In the included trials, levels of acceptance were generally modest, and adherence to the various protocols for wearing hip protectors was poor. Gillespie et al conclude that 'poor acceptance and adherence by older people offered hip protectors have been key factors contributing to the continuing uncertainty' of their effectiveness. ${ }^{1}$

Summaries of 12 economic evaluations, two of which were associated with two of the included trials, are also presented in the review. ${ }^{1}$ Gillespie et al note that the optimistic findings of older studies that the provision of hip protectors would be cost effective have been superseded by the more cautious conclusions of the more recent modelling studies.

On the basis of the review findings, Gillespie et al indicate the need for future trials to resolve the uncertainty about the effectiveness of providing and wearing hip protectors. They urge that the research community adopt the recommendations of the International Hip Protector Research Group. ${ }^{3}$ These include the focus on study populations, such as those in many nursing homes, at high risk of hip fracture (annual incidence of at least 3\%).

Together with those of over 4200 other Cochrane reviews, the full version of this review is available in The Cochrane Library (http://www.thecochranelibrary.com/). The review highlighted in this article is registered with the Cochrane, Bone, Joint and Muscle Trauma Group (http://www.bjmtg.cochrane.org/). The work of this Group involves preparing, maintaining and promoting the accessibility of systematic reviews on different aspects of the prevention, treatment, and rehabilitation of musculoskeletal injuries. People interested in contributing to this work can contact Lindsey Elstub, the Managing Editor, at lindsey.elstub@manchester.ac.uk.

\section{H Handoll}

Correspondence to $\mathrm{H}$ Handoll, Health and Social Care Institute, School of Health and Social Care, Teesside University, Middlesbrough TS1 3BA, UK; h.handoll@tees.ac.uk

Competing interests None.

Provenance and peer review Not commissioned; not externally peer reviewed.

Published Online First 11 November 2010

Injury Prevention 2010;16:431. doi:10.1136/ip.2010.030197

\section{REFERENCES}

1. Gillespie WJ, Gillespie LD, Parker MJ. Hip protectors for preventing hip fractures in older people. Cochrane Database Syst Rev 2010;(10):CD001255.

2. Kurrle SE, Cameron ID, Quine $S$, et al. Adherence with hip protectors: a proposal for standardised definitions. Osteoporos Int 2004;15:1-4.

3. Cameron ID, Robinovitch S, Birge $S$, et al. Hip protectors: recommendations for conducting clinical trials-an international consensus statement (part II). Osteopor Int 2010;21:1-10. 